\title{
Oncogenic function of angiopoietin-2 in vitro and its modulation of tumor progression in colorectal carcinoma
}

\author{
HYUNGJOO KIM ${ }^{1}$, TAE SUNG AHN ${ }^{2}$, CHANG-JIN KIM ${ }^{3}$, SANG BYUNG BAE ${ }^{4}$, \\ HAN JO KIM ${ }^{4}$, CHANG-SEUK LEE ${ }^{5}$, TAE HYUN KIM ${ }^{5}$, JUNGKYUN IM ${ }^{6}$, SANG HUN LEE ${ }^{7}$, \\ MYOUNG WON SON ${ }^{2}$, MOON SOO LEE ${ }^{2}$, MOO JUN BAEK $^{2}$ and DONGJUN JEONG ${ }^{3}$ \\ ${ }^{1}$ Soonchunhyang Medical Science Research Institute; Departments of ${ }^{2}$ Surgery, ${ }^{3}$ Pathology and ${ }^{4}$ Oncology, \\ College of Medicine, Soonchunhyang University, Dongnam-gu, Cheonan, Chungcheongnam-do 330-721; \\ Departments of ${ }^{5}$ Chemistry and ${ }^{6}$ Nanochemical Engineering, Soonchunhyang University, Shinchang-myeon, \\ Asansi, Chungcheongnam-do 336-745; ${ }^{7}$ Department of Biochemistry, College of Medicine, Soonchunhyang \\ University, Dongnam-gu, Cheonan, Chungcheongnam-do 330-721, Republic of Korea
}

Received August 31, 2015; Accepted January 26, 2017

DOI: $10.3892 / \mathrm{ol} .2017 .6203$

\begin{abstract}
Angiopoietin-2 (Ang-2) has been investigated in cancer primarily in terms of its angiogenic function, and its role as an oncogene has yet to be elucidated. The current study hypothesized that Ang-2 may be an oncogene and have a function in tumor progression. An investigation of the function of Ang-2 in the LoVo colorectal cancer (CRC) cell line in vitro, which expresses a high level of Ang-2, was performed by knocking down endogenous expression with a targeted short hairpin RNA. The aggressive phenotypic effects of Ang-2 on experimental and control group cells were assessed using cell proliferation, migration and invasion assays. The association between Ang-2 expression levels and clinicopathological factors was evaluated in 415 CRC tissues using immunohistochemistry. Suppressing Ang-2 expression decreased cellular proliferation, invasion and migration in an in vitro study. Ang-2 overexpression was observed in $46 \%$ of patients with CRC and was significantly associated with $\mathrm{pT}(\mathrm{P}=0.048), \mathrm{pN}(\mathrm{P}<0.001)$, venous invasion $(\mathrm{P}=0.023)$, lymphatic invasion $(\mathrm{P}<0.001)$ and tumor-node-metastasis stage $(\mathrm{P}=0.022)$. Furthermore, Ang-2 overexpression was an independent prognostic factor in $\mathrm{pN}$ stages 1 and 2. These results reveal that Ang-2 may be an oncogene in colorectal carcinogenesis and its expression may exert aggressive phenotypic effects during tumor progression. In addition, Ang-2 expression may serve as a prognostic marker and a potential drug target.
\end{abstract}

Correspondence to: Dr Dongjun Jeong, Department of Pathology, College of Medicine, Soonchunhyang University, 31 Soonchunhyang 6 gil, Dongnam-gu, Cheonan, Chungcheongnam-do 330-721, Republic of Korea

E-mail: juny1024@sch.ac.kr

Key words: Angiopoietin-2, Colorectal carcinoma, shRNA, Oncogenic function

\section{Introduction}

Angiopoietin (Ang)-1 and -2 are ligands of the Tie-2 receptor, which is a receptor tyrosine kinase expressed by endothelial cells during vascular development. Ang-1 stabilizes the mature vasculature by promoting the recruitment of pericytes and smooth muscle cells. By contrast, Ang-2 destabilizes the vasculature so that it assumes a flexible state more amenable to sprouting under the influence of the angiogenic inducer vascular endothelial growth factor (VEGF) (1). Ang-1 participates in the regulation of angiogenesis and lymphangiogenesis by competing with Ang-2 in binding to the Tie-2 receptor, which blocks Ang-1-mediated receptor phosphorylation and vascular maturation and stabilization (1). However, this function may be context-dependent; Ang-2 may induce endothelial cell apoptosis with consequent vascular regression by loosening cell-matrix contacts in the absence of VEGF while stimulating endothelial cell migration and proliferation, thereby serving as a proangiogenic signal for tumors in cooperation with VEGF and Ang-1 through Tie-2-dependent and/or -independent signaling pathways (2). In addition, Ang-2 is upregulated in various types of tumor, including breast (3), non-small cell lung (4), and gastric cancer (5), as well as hepatocellular carcinoma (6) and melanoma (7).

Colorectal cancer (CRC) is the third most common malignancy and the fourth highest cause of cancer mortality worldwide (8). It had the second highest incidence in 2010 and the highest rate of increase among cancer types in South Korea (9). CRC progression may lead to the dissemination of tumor cells to other tissues including the bone, brain, liver and lungs, where new tumors may arise. As metastatic colon cancer is associated with high mortality $(10,11)$, progression to metastasis is a turning point for CRC development, which depends on an angiogenic network (12). Although anti-metastatic molecule-based therapies, including the use of monoclonal antibodies, have been widely investigated (13), numerous patients with CRC still succumb to disease recurrence and metastasis. Therefore, insight into the association 
between the expression of angiogenic molecules and clinicopathological factors relating to the progression and prognosis of CRC is essential for developing novel therapeutic strategies to treat this disease.

Among the various studies investigating the expression of Ang-2 and its role in $\mathrm{CRC}$, research has primarily focused on tumor angiogenesis (14), and the link between Ang-2 expression and the biological characteristics of CRC has seldom been investigated. The oncogenic function of Ang-2 has not yet been studied and it remains to be elucidated. It was previously revealed that Ang-2 overexpression is associated with the invasive phenotype of CRC cells in vitro (15). The clinical significance of Ang-2 expression in CRC and its effects on tumor growth remain unclear. The current study investigated the malignant biological functions of Ang-2 as an oncogene using short hairpin (sh) RNA knockdown of the endogenous transcript in the LoVo colon cancer cell line. Furthermore, endogenous Ang-2 expression in CRC tumor cells was correlated with clinicopathological parameters. The in vitro results demonstrate that Ang-2 may be an oncogene, and its expression may serve as a prognostic marker and a potential therapeutic target for CRC.

\section{Materials and methods}

Tissue specimens and patient information. A total of 415 CRC tissue specimens containing tumor and adjacent normal tissue were collected from patients who were diagnosed and underwent colectomy or Miles' operation at Soonchunhyang University Cheonan Hospital (Cheonan, Korea) between January 2002 and December 2009. Specimens obtained from surgical resection were fixed in $10 \%$ neutral buffered formalin for $24 \mathrm{~h}$ at RT prior to being processed in paraffin. Patients did not receive any treatment prior to surgery. Patient data are presented in Table I. Informed consent was provided by all patients and the study protocol was approved by the Ethics Committee of Soonchunhyang University Cheonan Hospital.

Tissue microarray construction and immunohistochemistry. Tissue microarrays were produced from formalin-fixed, paraffin-embedded (FFPE) CRC blocks. Two cores of FFPE CRC block ( $2 \mathrm{~mm}$ in diameter) were embedded in a recipient block using a 2-mm puncher (Unitech Science, Seoul, South Korea). The paraffin tissue microarray (TMA) blocks were sectioned at $4-\mu \mathrm{m}$ thickness and transferred on the glass slide. The slides were baked for $1 \mathrm{~h}$ at $60^{\circ} \mathrm{C}$, deparaffinized with $100 \%$ zylene at 3 times for $5 \mathrm{~min}$, hydrated in gradient ethanol $(100-70 \%)$ and washed in $0.01 \mathrm{M}$ PBS at $\mathrm{pH} 7.2 \pm 7.4$. The antigens were retrieved by heating the slides in $0.01 \mathrm{M}$ citrate buffer (pH 6.0, Sigma-Aldrich; Merck Millipore, Darmstadt, Germany) in a pressure cooker for $15 \mathrm{~min}$. Sections were incubated overnight at $4^{\circ} \mathrm{C}$ with human Ang-2 polyclonal antibody (dilution, 1:20; cat. no. GTX100991, GeneTex, Inc., Irvine, CA, USA). Incubation with secondary antibody was performed using an EnVision Horseradish Peroxidase-Labeled Polymer (cat. no. K400311, Dako; Agilent Technologies, Inc., Santa Clara, CA, USA) for $30 \mathrm{~min}$, at room temperature followed by treatment with $100 \mu 1$ 3,3'-diaminobenzidene (Dako; Agilent Technologies, Inc.). The TMA slides were counterstained with Harris's hematoxylin for $20 \mathrm{sec}$, dehydrated in gradient ethanol (80-100\%), cleared with $100 \%$ zylene and mounted with Canada balsam (cat. no. C1795, Sigma-Aldrich; Merck Millipore). Immunostaining was scored by counting 5 random fields using a light microscope for each stained tissue section according to a semi-quantitative optical analysis by three independent investigators blinded to the clinical data, and a consensus score was determined for each specimen.

Immunohistochemistry data analysis. The expression of Ang-2 was assessed in terms of staining intensity and the percentage of positive cells by light microscopy, as previously described (7). Staining intensity was scored as follows: 0 ) no detectable staining; 1) faint staining; 2) moderate staining; $3)$ intense staining. The percentage of positive cells was also classified as one of four categories: 0) $(0 \%), 1)(1-10 \%)$, 2) (11-50\%), and 3) (51-100\%). The final score, calculated as the product of the intensity and percentage scores, was grouped into low and high expression (low; scores $\leq 3$ and high; scores $\geq 4$ ).

Cell lines and culture. Five human CRC cell lines (HCT116, SW480, LoVo, HT29 and colo205) were obtained from the Korean cell line bank (Seoul, Korea). The CCD841 CoN normal colon epithelial cells and HUVEC cells were obtained from the American Type Culture Collection (Manassas, VA, USA). The CRC cell lines (HCT116, SW480, LoVo, HT29 and Colo205) were screened to select a high Ang-2-expressing line. The CCD841 CoN normal colon epithelial cell line was used as Ang-2 expressing negative control and the vascular endothelial HUVEC cell line was used as Ang-2 expressing positive control. Cells were cultured in RPMI 1640 medium supplemented with $10 \%$ fetal bovine serum and $1 \%$ penicillin-streptomycin (all from Gibco; Thermo Fisher Scientific, Inc., Waltham, MA, USA) at $37^{\circ} \mathrm{C}$ in an atmosphere containing $5 \% \mathrm{CO}_{2}$. Ang-2 expression levels were evaluated by reverse transcription-polymerase chain reaction (RT-PCR) and western blot analysis. LoVo cells expressed the highest levels of Ang-2 and were therefore used in the following assays.

RT-PCR. CRC HCT116, SW480, LoVo, HT29, Colo205, CCD-841, and HUVEC cell lines were lysed using $1 \mathrm{ml}$ of QIAzol in the RNeasy kit (Qiagen, Inc., Valencia, CA, USA) to extract RNAs. The total RNA of shRNA-transfected LoVo cells was extracted 4 days after transfection. A total of $1 \mu \mathrm{g}$ RNA was reverse transcribed using the ReverTra Ace qPCR kit (Toyobo Co., Ltd., Osaka, Japan). Semi-quantitative PCR was performed using the Maxime PCR PreMix kit (Intron Biotechnology, Inc., Sungnam, Korea) and the following primer pairs: Ang-2 forward, 5'-GGATCTGGGGAGAGA GGAAC-3'; reverse, 5'-CTCTGCACCGAGTCATCGTA-3'; GAPDH forward, 5'-ACCACTTTGTCAAGCTCATT-3' and reverse, 5'-AGGAAGAGAGAGACCCTCAC-3'. PCR amplification of Ang-2 and GAPDH was performed in 35 cycles (Ang-2) or 38 cycles (GAPDH) as follows: $30 \mathrm{sec}$ of denaturing at $95^{\circ} \mathrm{C}, 60 \mathrm{sec}(\mathrm{Ang}-2)$ or $30 \mathrm{sec}(\mathrm{GAPDH})$ of annealing at $56^{\circ} \mathrm{C}$ (Ang-2) and $58^{\circ} \mathrm{C}(\mathrm{GAPDH})$, and $30 \mathrm{sec}$ of extension at $72^{\circ} \mathrm{C}$. PCR products were analyzed and visualized by capillary electrophoresis (Qiagen, Inc.). 
Table I. Comparison of clinicopathological factors and Ang-2 expression.

\begin{tabular}{|c|c|c|c|c|}
\hline \multirow{2}{*}{$\begin{array}{l}\text { Clinicopathological } \\
\text { factors }\end{array}$} & \multicolumn{2}{|c|}{ Ang-2 } & \multirow{2}{*}{$\begin{array}{c}\text { Total } \\
(\mathrm{N}=415)\end{array}$} & \multirow[b]{2}{*}{ P-value } \\
\hline & High Exp. $(\mathrm{N}=190)$ & Low Exp. $(\mathrm{N}=225)$ & & \\
\hline Age, years (SD) & $63.3 \pm 12.9$ & $62.4 \pm 12.3$ & $62.8 \pm 12.6$ & 0.488 \\
\hline Sex, N (\%) & & & & 0.764 \\
\hline Male & $107(56.3)$ & $130(57.8)$ & $237(57.1)$ & \\
\hline Female & $83(43.7)$ & $95(42.2)$ & $178(42.9)$ & \\
\hline pT stage, N (\%) & & & & 0.048 \\
\hline 1 & $7(3.7)$ & $14(6.4)$ & $21(5.1)$ & \\
\hline 2 & $25(13.2)$ & $38(16.9)$ & $63(15.2)$ & \\
\hline 3 & $122(64.2)$ & $148(65.8)$ & $270(65.1)$ & \\
\hline 4 & $36(18.9)$ & $25(11.1)$ & $61(14.7)$ & \\
\hline pN stage, $\mathrm{N}(\%)$ & & & & $<0.001$ \\
\hline 0 & $86(45.3)$ & $140(62.2)$ & $226(54.5)$ & \\
\hline 1 & $56(29.5)$ & $63(28.0)$ & $119(28.7)$ & \\
\hline 2 & $48(25.3)$ & $22(9.8)$ & $70(16.9)$ & \\
\hline Venous invasion & & & & 0.023 \\
\hline 0 & $142(74.7)$ & $187(83.1)$ & $329(79.3)$ & \\
\hline 1 & $48(25.3)$ & $38(16.9)$ & $86(20.7)$ & \\
\hline Lymphatic invasion & & & & $<0.001$ \\
\hline 0 & 117 (61.6) & $184(81.8)$ & $301(72.5)$ & \\
\hline 1 & $73(38.4)$ & $41(18.2)$ & $114(27.5)$ & \\
\hline TNM stage & & & & 0.022 \\
\hline $\mathrm{I}$ & $24(12.6)$ & $38(16.9)$ & $62(14.9)$ & \\
\hline II & $63(33.2)$ & $98(43.6)$ & $161(38.8)$ & \\
\hline III & $91(47.9)$ & $82(36.4)$ & $173(41.7)$ & \\
\hline IV & $12(6.3)$ & $7(3.1)$ & $19(4.6)$ & \\
\hline
\end{tabular}

Exp., expression; SD, standard deviation; TNM, tumor-node-metastasis; Ang-2, Angiopoietin-2.

Western blotting. CRC HCT116, SW480, LoVo and HT29 cells were collected in buffer $(62.5 \mathrm{mmol} / \mathrm{l}$ Tris- $\mathrm{HCl}, \mathrm{pH} 6.8$; $10 \%$ glycerol; $2 \%$ sodium dodecyl sulfate) and heated for $10 \mathrm{~min}$ at $100^{\circ} \mathrm{C}$. Protein concentration was determined by the bicinchoninic acid assay using an xMark Microplate Absorbance Spectrophotometer (Bio-Rad Laboratories, Inc., Hercules, CA, USA). Equal quantities $(30 \mu \mathrm{g} / 20 \mu \mathrm{l})$ of protein were separated by electrophoresis on Mini-PROTEAN TGX precast gels (Bio-Rad Laboratories, Inc.), using $\beta$-actin as a loading control, and were transferred onto an Immobilon polyvinylidene difluoride membrane (Millipore, Billerica, MA, USA), which was probed with anti-human Ang-2 polyclonal antibody (dilution, 1:2,000; GeneTex, Inc.). The signal was detected using an enhanced chemiluminescence kit (Advansta, Menlo Park, CA, USA) and a Molecular Imager ChemiDoc XRS+ system (Bio-Rad Laboratories, Inc.).

RNA interference. LoVo cells $\left(1 \times 10^{6}\right)$ were grown overnight in RPMI 1640 medium in six-well culture plates to $60-70 \%$ confluence prior to transfection with Ang-2 shRNA lentiviral particles (TRCN000005923, TRCN000005924, TRCN000005925, TRCN000005926, and TRCN000005927), which were purchased from
Sigma-Aldrich (Merck Millipore) along with a non-target negative control shRNA (SHC002V). The negative controls consisted of shRNA untransfected LoVo cells and non-target negative control shRNA (HSC002V) transfected LoVo cells. Lentiviral transfection was performed according to the manufacturer's protocol. Transfected cells were cultured for 2 additional days prior to puromycin selection $(1 \mathrm{mg} / \mathrm{ml}$; Sigma-Aldrich; Merck Millipore). Ang-2 levels were determined by RT-PCR and western blotting.

Cell proliferation assay. The negative controls and Ang2-target shRNA transfected LoVo cells were seeded (1x10 4 cells/well) in 96-well culture plates and incubated for $48 \mathrm{~h}$. An MTT assay was performed by adding $10 \mu \mathrm{l}$ MTT ( $5 \mathrm{mg} / \mathrm{ml}$; Sigma-Aldrich; Merck Millipore) to each well prior to incubation for $4 \mathrm{~h}$ at $37^{\circ} \mathrm{C}$, followed by the addition of $100 \mu \mathrm{l}$ dimethyl sulfoxide to each well prior to overnight incubation. The following day, cell proliferation/viability was measured as the absorbance at $570 \mathrm{~nm}$.

Invasion assay. The negative controls and Ang2-target shRNA transfected LoVo cells were cultured for $48 \mathrm{~h}$ in serum-free RPMI 1640 with penicillin-streptomycin $(5 \mathrm{U} / \mathrm{ml})$; 


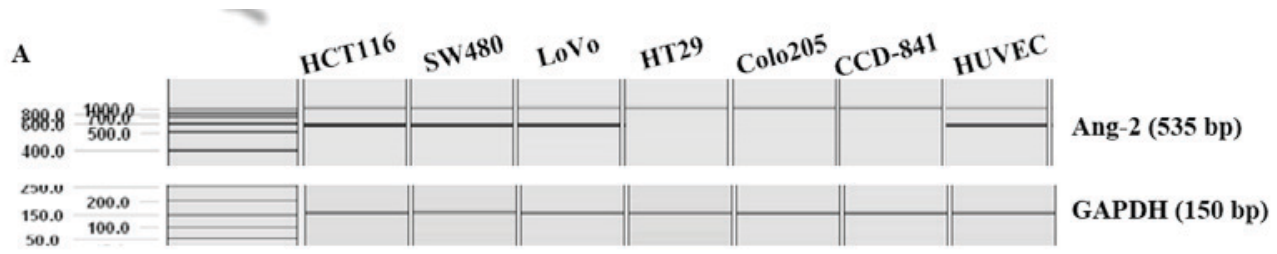

B
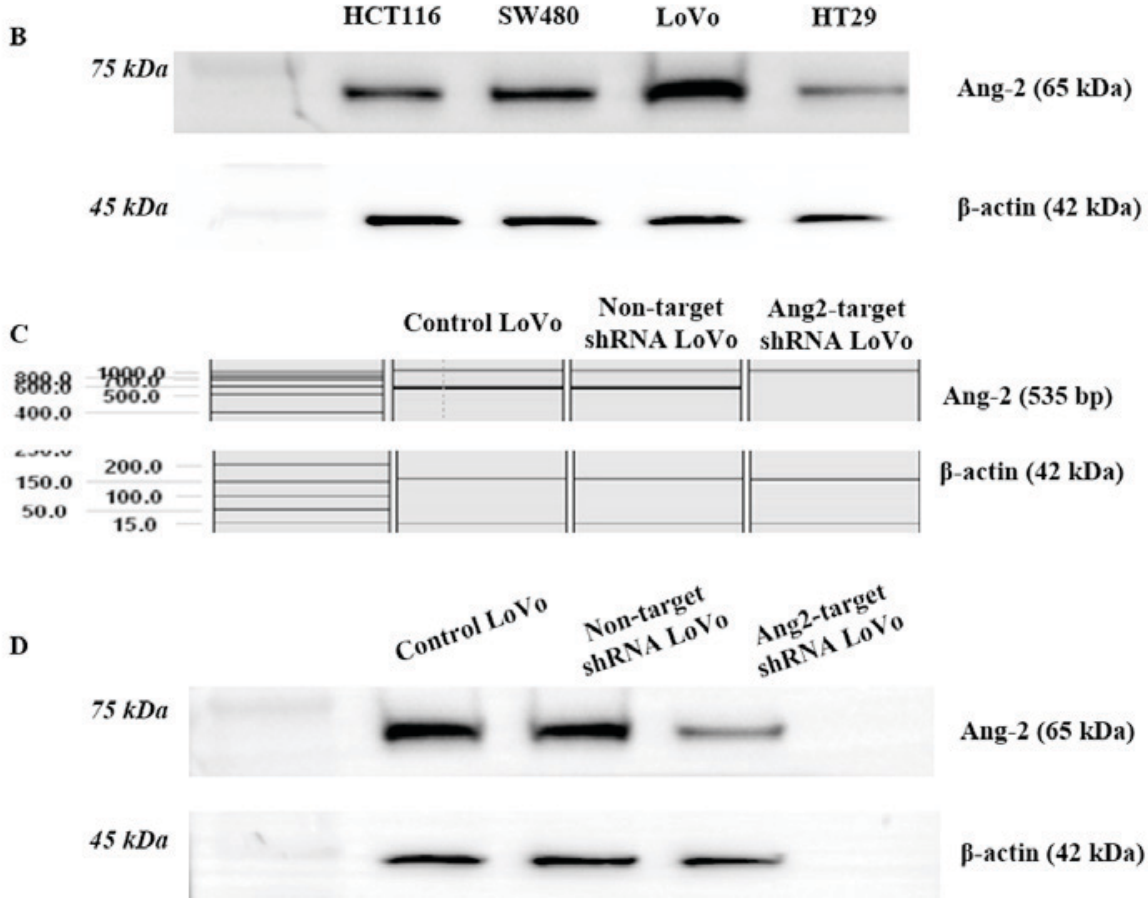

Figure 1. Ang-2 expression was investigated by reverse-transcription polymerase chain reaction and western blot analysis in colorectal cancer cell lines, HUVEC and normal cell line (CCD-841 CoN). (A) Among 5 colorectal cancer cell lines, 3 cell lines expressed Ang-2 mRNA, as did the HUVEC cell line. (B) LoVo cells expressed a markedly higher level of Ang-2 protein compared with other colorectal cancer cell lines, as detected by western blotting. (C) The LoVo cell line transfected with Ang-2 target shRNA did not express Ang-2 mRNA. (D) The LoVo cell transfected with Ang-2 target shRNA exhibited markedly decreased expression levels of Ang-2 protein. Ang-2, Angiopoietin-2.

6.5-mm polycarbonate filters ( 8 - $\mu$ m pore size,EMD Millipore, Billerica, MA, USA) were coated with growth factor-reduced Matrigel (BD Biosciences, San Jose, CA, USA) and incubated

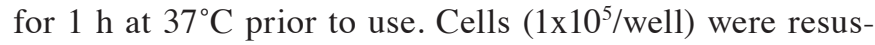
pended in the top chamber while $700 \mu 1$ culture medium with $10 \%$ FBS was added to the bottom chamber as a chemoattractant. After $48 \mathrm{~h}$ incubation at $37^{\circ} \mathrm{C}$, the medium in the bottom chamber was removed, and cells were fixed with $3.5 \%$ paraformaldehyde and their nuclei stained with methyl green.

The number of cells that had migrated through the pores of the filter and into the bottom chamber was counted under a phase contrast microscope. Cells in five random visual fields at x20 magnification were counted for each well and used to determine an average value. The experiment was performed in triplicate.

Cell migration assay. A wound healing cell migration assay was performed using the 24-well cell culture insert system (Ibidi GmbH, München, Germany). The negative controls or Ang2-target shRNA transfected LoVo cells were resuspended in RPMI 1640 containing 10\% FBS and were seeded $\left(1 \times 10^{5}\right.$ cells) into each well of the insert. After $48 \mathrm{~h}$, the inserts were removed and cells were cultured in fresh medium. Images were captured using a phase contrast microscope with an AxioCam camera (Zeiss, Jena, Germany). The size of the cell-free gap was monitored for 12, 24 and $48 \mathrm{~h}$ and measured in five random visual fields to determine an average value.

Statistical analysis. Data were analyzed using SPSS version 18.0 (SPSS, Inc., Chicago, IL, USA). The clinical and pathological factors that were evaluated included primary tumors (pT; 1, 2, 3 and 4$)$, primary nodes (pN; 0,1 and 2), vascular and lymphatic invasion and TNM stage classification (I, II, III and IV). The association between Ang-2 expression and clinicopathological factors was evaluated with the $\chi^{2}$ test and a two-sided t-test. A survival analysis using the Kaplan-Meier method was performed based on clinical and pathological variables and Ang-2 expression, and the statistical significance at $\mathrm{P}<0.05$ was assessed by the log-rank test. A multivariate Cox regression analysis in which all covariates were controlled was performed to evaluate the association between clinicopathological factors and Ang-2 expression, with hazard ratios (HR) and 95\% confidence intervals (CI) estimated from Cox proportional hazard models. $\mathrm{P}<0.05$ was considered to indicate a statistically significant difference. 

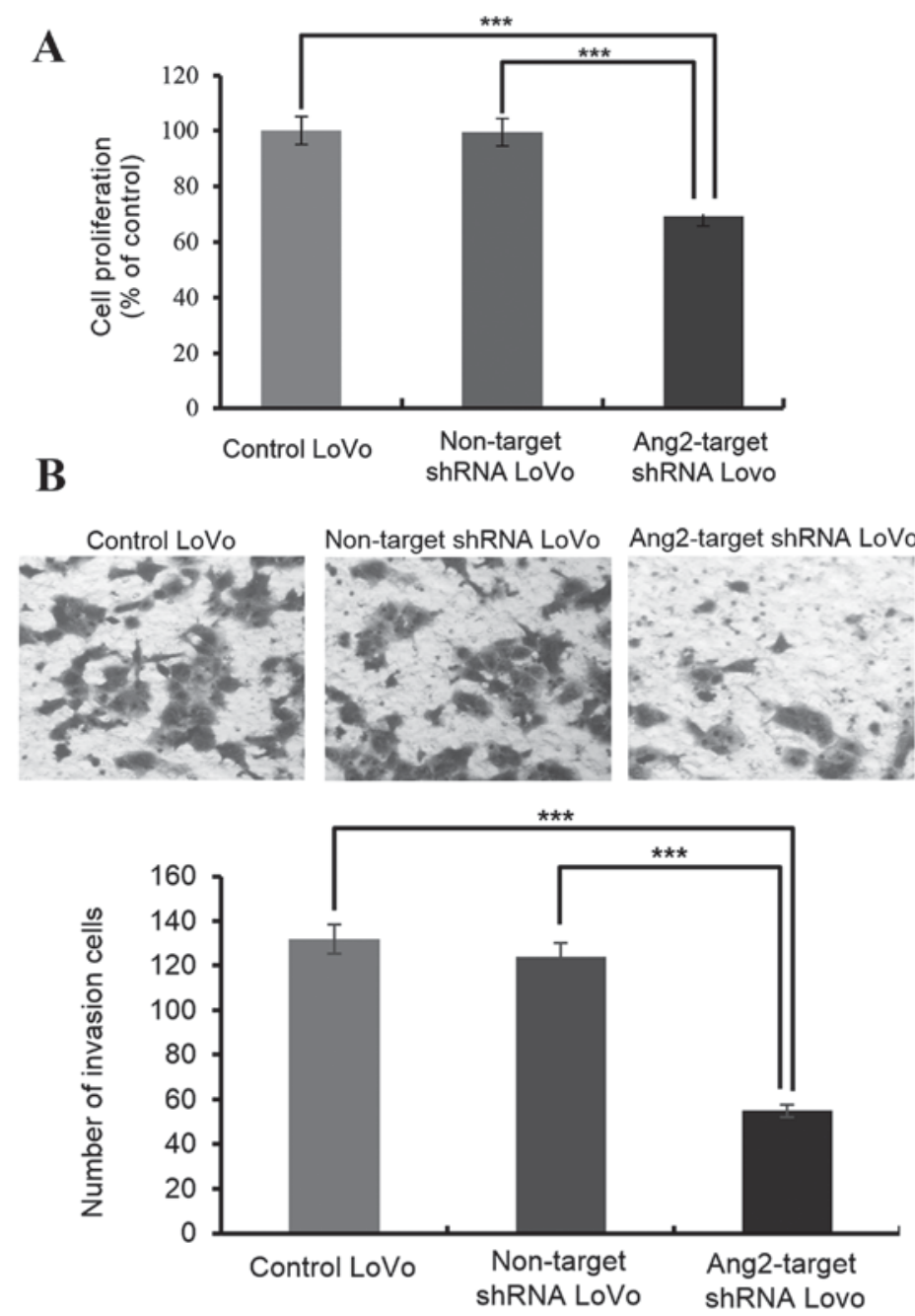

Figure 2. (A) The proliferation of LoVo cells following Ang-2-targeted shRNA transfection was significantly decreased compared with control LoVo cells and LoVo cell transfected with non-targeted shRNA. (B) The invasion ability of Ang-2 target shRNA transfected LoVo cells was decreased markedly compared with the control and non-target shRNA-transfected LoVo cells. ${ }^{* * *} \mathrm{P}<0.05$. Ang-2, Angiopoietin-2; shRNA, short hairpin RNA.

\section{Results}

Ang-2 is expressed in CRC cell lines and HUVECs. HUVECs expressed a high level of Ang-2 and were used as a positive control. Three of the five colorectal cancer cell lines screened (HCT116, SW480 and LoVo) had Ang-2 levels that were comparable with those of HUVECs, whereas the CCD-841CoN cell line did not express Ang-2 (Fig. 1A). LoVo cells had the highest Ang-2 level as determined by western blot analysis (Fig. 1B) and they were used for shRNA knockdown studies. The shRNA-mediated knockdown of Ang-2 in LoVo cells was confirmed by RT-PCR (Fig. 1C) and by western blotting (Fig. 1D). The Ang-2 transcript and protein were detected in untransfected control LoVo cells and non-target shRNA transfected LoVo cells, but they were not expressed in Ang-2-target shRNA transfected LoVo cells, indicating that endogenous Ang-2 expression was effectively suppressed (Fig. 1C and D).

Ang-2 knockdown leads to the loss of malignant features in CRC cells. Malignant cells are characterized by the capacity to proliferate, migrate and invade tissues. Ang-2 knockdown resulted in a $35 \%$ decrease in cell proliferation compared with untransfected controls or non-target shRNA transfected LoVo cells, as determined by the MTT assay (Fig. 2A). Similarly, cell invasion was markedly decreased by Ang-2 knockdown (Fig. 2B). Furthermore, compared with control cells, the cell migration at all the time points examined (12,24 and $48 \mathrm{~h}$ ) was reduced upon knockdown of Ang-2 (Fig. 3).

Ang-2 overexpression is associated with clinical features of $C R C$. Ang-2 expression in tissue specimens from patients with CRC was examined by immunohistochemistry. Ang-2 was rarely observed in normal colorectal epithelial cells but was intensely stained in the cytoplasm of tumor cells (Fig. 4A). Elevated Ang-2 expression was observed in 190/415 cases (46\%) of CRC. The association between Ang-2 expression and clinicopathological parameters of 415 patients with $\mathrm{CRC}$ is presented in Table I. Although there were no correlations with patient age or sex, Ang-2 expression was associated with pT and $\mathrm{pN}$ stages $(\mathrm{P}<0.05$ and $\mathrm{P}<0.001$, respectively), venous and lymphatic invasion $(\mathrm{P}=0.023$ and $\mathrm{P}<0.001$, respectively), and clinical stage $(\mathrm{P}=0.022)$.

The multivariate Cox proportional hazards regression analysis revealed that $\mathrm{pN}$ stages 1 and 2 were significantly 

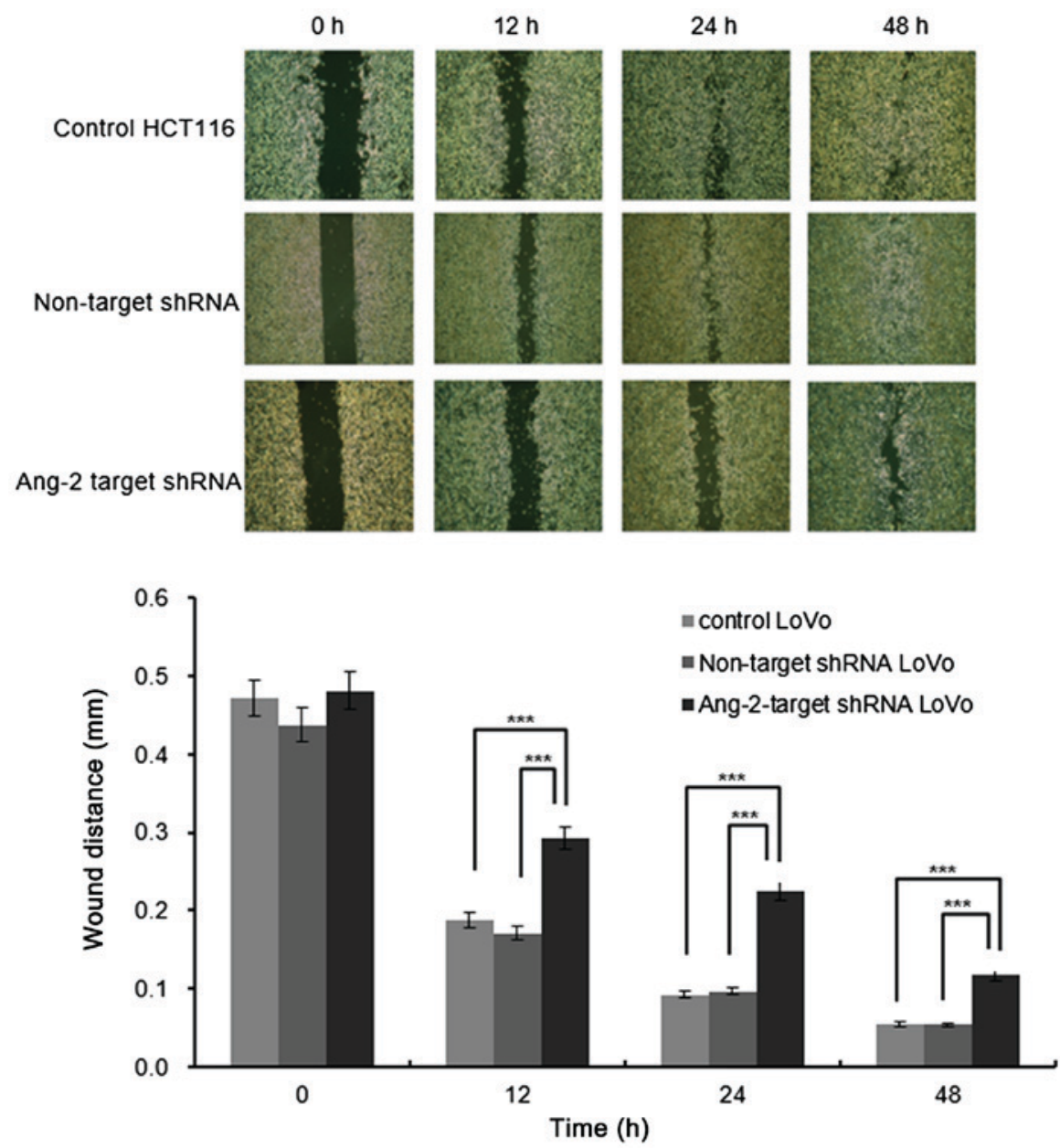

Figure 3. The migration of Ang-2 target shRNA transfected LoVo cells was decreased compared with the control and non-target shRNA transfected LoVo cells. ${ }^{* * *} \mathrm{P}<0.01$. Ang-2, Angiopoietin-2; shRNA, short hairpin RNA.
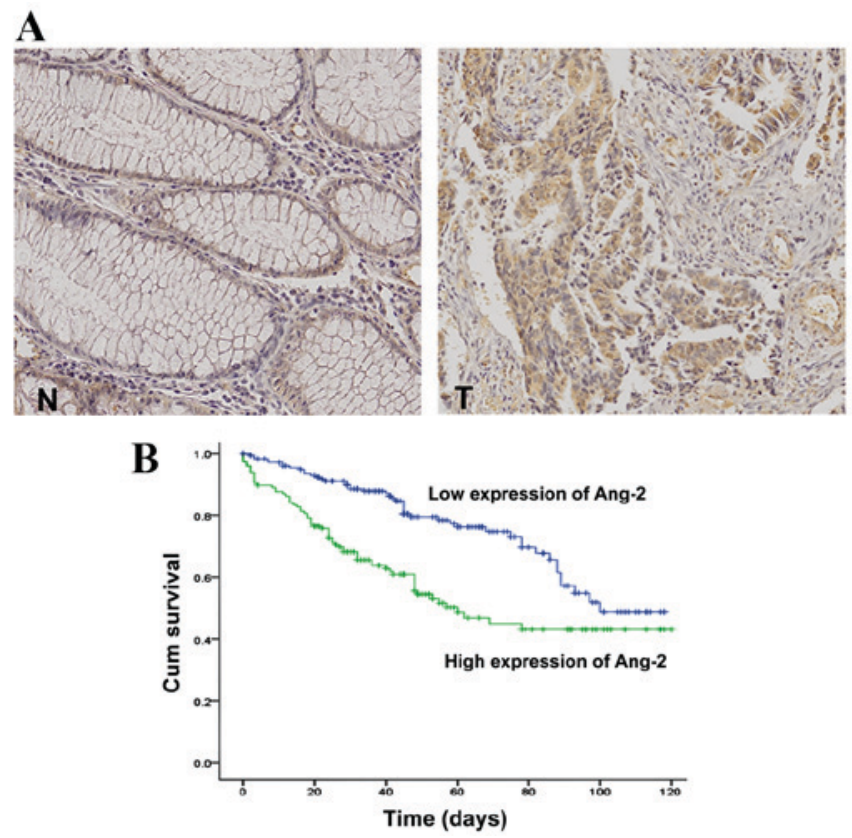

Figure 4. (A) Ang-2 was not expressed in N cells. However, Ang-2 expression was markedly increased in T cells. (B) The cumulative survival was significantly decreased in patients with high levels of Ang-2 compared with patients with low levels of Ang-2 expression, as determined by Kaplan Meier analysis (log rank test, $\mathrm{P}<0.001)$. T, colorectal cancer cells; $\mathrm{N}$, normal colonic epithelial cells; Cum, cumulative; Ang-2, Angiopoietin-2. association with high mortality, with HRs of 5.17 (95\% CI, 1.36-19.59; $\mathrm{P}=0.016)$ and 7.01 (95\% CI, 1.76-27.96; $\mathrm{P}=0.006)$, respectively. Furthermore, Ang-2 expression in patients with CRC was associated with high mortality, with an HR of 6.78 (95\% CI, 4.16-11.05; $\mathrm{P}<0.001$ ), indicating that Ang-2 expression is an independent prognostic factor for CRC progression (Table II). The mean survival time was markedly diminished in patients with high Ang-2 expression levels compared with those with low Ang-2 expression levels ( $\mathrm{P}=0.001$; Fig. 4B).

\section{Discussion}

Rapidly proliferating tumor cells require a greater oxygen supply than normal cells, creating a relatively hypoxic microenvironment surrounding the tumor; this triggers the secretion of hypoxia-inducible factors from endothelial or tumor cells that induce cytokines, such as angiopoietin, VEGF, and Tie (16-19). Constitutive, low-level expression of Ang-1 by normal tissue stabilizes existing blood vessels, whereas Ang-2 overexpression in newly formed tumors leads to vessel destabilization and hypoxia; therefore, cancer progression depends on the dysregulation of the balance between normal and pathological neoangiogenesis (14).

The current study revealed that endogenous Ang-2 expression in cancer cell lines is associated with its aggressive 
Table II. Cox regression analysis of clinicopathological factors.

\begin{tabular}{|c|c|c|}
\hline $\begin{array}{l}\text { Clinicopathological } \\
\text { factors }\end{array}$ & $\begin{array}{l}\text { Hazard ratio } \\
(95 \% \mathrm{CI})\end{array}$ & $\mathrm{P}$-value \\
\hline Age, years & $1.03(1.01-1.04)$ & 0.003 \\
\hline \multicolumn{3}{|l|}{ Sex } \\
\hline \multicolumn{3}{|l|}{ Female } \\
\hline Male & $0.90(0.62-1.31)$ & 0.569 \\
\hline pT stage & & 0.291 \\
\hline 1 & 1.00 & \\
\hline 2 & $0.67(0.14-3.19)$ & 0.618 \\
\hline 3 & $0.46(0.09-2.50)$ & 0.371 \\
\hline 4 & $0.77(0.13-4.47)$ & 0.770 \\
\hline \multicolumn{3}{|l|}{ pN stage } \\
\hline 0 & 1.00 & \\
\hline 1 & $5.17(1.36-19.59)$ & 0.016 \\
\hline 2 & $7.01(1.76-27.96)$ & 0.006 \\
\hline \multicolumn{3}{|l|}{ Venous invasion } \\
\hline \multicolumn{3}{|l|}{0} \\
\hline 1 & $1.27(0.72-2.26)$ & 0.410 \\
\hline \multicolumn{3}{|l|}{ Lymphatic invasion } \\
\hline \multicolumn{3}{|l|}{0} \\
\hline 1 & $0.90(0.52-1.57)$ & 0.719 \\
\hline \multicolumn{3}{|l|}{ TNM stage } \\
\hline $\mathrm{I}$ & 1.00 & \\
\hline II & $1.83(0.68-4.99)$ & 0.234 \\
\hline III & $0.43(0.12-1.59)$ & 0.206 \\
\hline IV & $1.24(0.31-5.00)$ & 0.761 \\
\hline \multicolumn{3}{|l|}{ Ang-2 expression } \\
\hline \multicolumn{3}{|l|}{0} \\
\hline 1 & $6.78(4.16-11.05)$ & $<0.001$ \\
\hline
\end{tabular}

CI, confidence interval; Ang-2, Angiopoietin-2; TNM, tumor-node-metastasis.

phenotype as a potential oncogene, including processes such as proliferation, migration and invasion. The inhibition of Ang-2 in the LoVo CRC cell line, which has a high endogenous level of Ang-2, resulted in decreased proliferation, migration and invasion, which may indicate that Ang-2 affects these aspects of the tumorigenic phenotype as a potential oncogene in CRC.

The tumor tissue is composed of various types of cell, including tumor parenchymal cells, stromal cells, immune inflammatory cells and vascular cells. The origin of Ang-2 in tumor tissue is not certain. Although early studies indicated that Ang-2 was primarily expressed in endothelial cells, other studies now point to its expression in various tumor parenchymal cells, including breast, gastric and lung cancer as well as melanoma and hepatocellular carcinoma (3-7); however, the role of Ang-2 in CRC is controversial. It has been reported that Ang-2 is almost exclusively produced by endothelial cells themselves, suggesting an autocrine mode of neoangiogenic action (20). One study reported high Ang-2 expression in tumor cells (21), whilst another reported high Ang-2 expression in the stromal compartment of CRC tissues but not in tumor cells (22).

In the current study, Ang-2 overexpression was significantly associated with an aggressive tumor phenotype, as evidenced by $\mathrm{pT}, \mathrm{pN}$ and TNM stages and venous and lymphatic invasion. Ang-2 overexpression in CRC was an independent prognostic factor for decreased survival in $\mathrm{pN}$ stages 1 and 2, which is concordant with the findings of a previous study, which demonstrated that the upregulation of Ang-2 expression in CRC was associated with lymph node metastasis (19).

Ang-2 inhibition decreases tumor size as well as lumen formation in tumor-associated vessels in vivo (20). Although the precise underlying mechanism of Ang-2 function in CRC remains unknown, in triple-negative breast cancer Ang-2 secreted by tumor cells breaches the blood brain barrier, leading to brain metastasis (23). Ang-2 promotes metastasis by inducing epithelial-mesenchymal transition through $\beta 1$ integrin-mediated signaling. Structurally, Ang-2 has a highly conserved $\mathrm{N}$-terminal fibrinogen-like receptor-binding domain that may interact with specific integrin receptors (24). The association between Ang-2 expression and aggressive tumor phenotype has been studied in U87MG glioma cells, in which upregulation of Ang-2 expression leads to increased invasion (25).

To the best of our knowledge, this study is the first to report a major role for Ang-2 in tumor progression that involves stimulatory effects on angiogenesis through the paracrine function of Ang-2 secreted by tumor cells. It remains to be established whether Ang-2 is expressed at the early stages of the tumor. The growth rates of tumors derived from lung cancer and melanoma cell lines differ for wild-type and Ang-2-deficient mice during the early stages of tumor development, whereas the growth rates were similar during the later stages of primary tumor progression (26). However, in the present study, $83.2 \%$ of pT 3 and 4 cases exhibited Ang- 2 overexpression, and only $16.8 \%$ of pT1 and 2 cases had Ang-2 overexpression, which may indicate that Ang-2 expression exerts fewer effects in the early stages of colorectal cancer compared with the late stage as a function of tumor progression. This difference may be due to the variability of cancer tissues. In one previous study, Ang-2 expression was significantly upregulated in CRC and associated with lymph node metastases (27), consistent with the observations of the present study, in which Ang-2 overexpression was associated with TNM stages III and IV and lymph node metastasis.

The diverse roles of Ang-2 in tumor progression have been extensively studied. However, the focus has been on its paracrine effect on angiogenesis through a Tie2-dependent pathway in endothelial cells (28), rather than through its aberrant overexpression in tumor cells and autocrine effects, which is closely associated with tumor progression. For instance, in gastric cancer, endogenous Ang-2 expression in tumor cells has a critical role in tumor metastasis and is correlated with Ang-2 expression in endothelial cells (29).

In conclusion, the present study revealed that production of Ang-2, functioning as a potential oncogene in vitro, in cancer cell lines resulted in aggressive cell phenotypes. Ang-2 expression in tumor parenchymal cells may be a 
marker of CRC progression that is associated with the clinical outcome of patients. Given its involvement in a wide range of tumor-specific processes, Ang-2 is a potential therapeutic target for CRC treatment. Additional studies are underway to investigate the factors downstream of Ang-2 that are responsible for modulating these various cellular processes by promoting invasion and metastasis.

\section{Acknowledgements}

The present study was supported by the Soonchunhyang University Research Fund.

\section{References}

1. Fiedler U, Krissl T, Koidl S, Weiss C, Koblizek T, Deutsch U, Martiny-Baron G, Marmé D and Augustin HG: Angiopoietin-1 and angiopoietin-2 share the same binding domains in the Tie-2 receptor involving the first Ig-like loop and the epidermal growth factor-like repeats. J Biol Chem 278: 1721-1727, 2003.

2. Hu B and Cheng SY: Angiopoietin-2: Development of inhibitors for cancer therapy. Curr Oncol Rep 11: 111-116, 2009.

3. Rykala J, Przybylowska K, Majsterek I, Pasz-Walczak G, Sygut A, Dziki A and Kruk-Jeromin J: Angiogenesis markers quantification in breast cancer and their correlation with clinicopathological prognostic variables. Pathol Oncol Res 17: 809-817, 2011.

4. Zhang ZL, Liu ZS and Sun Q: Expression of angiopoietins, Tie2 and vascular endothelial growth factor in angiogenesis and progression of hepatocellular carcinoma. World J Gastroenterol 12: 4241-4245, 2006.

5. Tanaka F, Ishikawa S, Yanagihara K, Miyahara R, Kawano T, Li M, Otake Y and Wada H: Expression of angiopoietins and its clinical significance in non-small cell lung cancer. Cancer Res 62: 7124-7129, 2002.

6. Moom WS, Park HS, Yu KH, Jang KY, Kang MJ, Park H and Tarnawski AS: Expression of angiopoietin 1,2 and their common receptor Tie2 in human gastric carcinoma: Implication for angiogenesis. J Korean Med Sci 21: 272-278, 2006.

7. Helfrch I, Edler L, Sucker A, Thomas M, Christian S, Schadendorf D and Augustin HG: Angiopoietin-2 levels are associated with disease progression in metastatic malignant melanoma. Clin Cancer Res 15: 1384-1392, 2009.

8. Jemal A, Siegel R, Ward E, Hao Y, Xu J and Thun MJ: Cancer statistics, 2008. CA Cancer J Clin 58: 71-96, 2008.

9. Jung KW, Won YJ, Kong HJ, Oh CM, Seo HG and Lee JS: Cancer statistics in Korea: Incidence, mortality, survival and prevalence in 2010. Cancer Res Treat 45: 1-14, 2013.

10. Goldberg RM: Therapy for metastatic colorectal cancer. Oncologist 11: 981-987, 2006.

11. Haddad AJ, Bani Hani M, Pawlik TM and Cunningham SC: Colorectal liver metastases. Int J Surg Oncol 2011: 285840, 2011

12. Carmeliet P and Jain RK: Angiogenesis in cancer and other diseases. Nature 407: 249-257, 2000.

13. Scott AM, Wolchok JD and Old LJ: Antibody therapy of cancer. Nat Rev Cancer 12: 278-287, 2012.
14. Ahmad SA, Liu W, Jung YD, Fan F, Wilson M, Reinmuth N, ShaheenRM,BucanaCDandEllisLM:Theeffects of angiopoietin-1 and -2 on tumor growth and angiogenesis in human colon cancer. Cancer Res 61: 1255-1259, 2001.

15. Rigamonti N, Kadioglu E, Keklikoglou I, Wyser Rmili C, Leow CC and De Palma M: Role of angiopoietin-2 in adaptive tumor resistance to VEGF Signaling Blockade. Cell Rep 8: 696-706, 2014.

16. Rankin EB and Giaccia AJ: The role of hypoxia-inducible factors in tumorigenesis. Cell Death Differ 15: 678-685, 2008.

17. Peters KG: Vascular endothelial growth factor and the angiopoietins: Working together to build a better blood vessel. Circ Res 83: 342-343, 1998 .

18. Li C, Sun CJ, Fan JC, Geng N, Li CH, Liao J, Mi K, Zhu GQ, $\mathrm{Ma} \mathrm{H}$, Song YF, et al: Angiopoietin-2 expression is correlated with angiogenesis and overall survival in oral squamous cell carcinoma. Med Oncol 30: 571, 2013.

19. Ochiumi T, Tanaka S, Oka S, Hiyama T, Ito M, Kitadai Y, Haruma K and Chayama K: Clinical significance of angiopoietin-2 expression at the deepest invasive tumor site of advanced colorectal carcinoma. Int J Oncol 24: 539-547, 2004.

20. Scharpfenecker M, Fiedler U, Reiss Y and Augustin HG: The Tie-2 ligand angiopietin-2 destabilizes quiescent endothelium through an internal autocrine loop mechanism. J Cell Sci 15: 771-780, 2005.

21. Ahmad SA, Liu W, Jung YD, Fan F, Reinmuth N, Bucana CD and Ellis LM: Differential expression of angiopoietin-1 and angiopoietin-2 in colon carcinoma. A possible mechanism for the initiation of angiogenesis. Cancer 92: 1138-1143, 2001.

22. Kahlert C, Pecqueux M, Halama N, Dienemann $H$, Muley T, Pfannschmidt J, Lasitschka F, Klupp F, Schmidt T, Rahbari N, et al: Tumour-site-dependent expression profile of angiogenic factors in tumour-associated stroma of primary colorectal cancer and metastases. Br J Cancer 110: 441-449, 2014.

23. Avraham HK, Jiang S, Fu Y, Nakahatri H, Ovadia $\mathrm{H}$ and Avraham S: Angiopoietin-2 mediates blood-brain barrier impairment and colonization of triple-negative breast cancer cells in brain. J Pathol 232: 369-381, 2014

24. Shim WS, Ho IA and Wong PE: Angiopoietin: A TIE(d) balance in tumor angiogenesis. Mole Cancer Res 5: 655-665, 2007.

25. Hu B, Jarzynka MJ, Guo P, Imanishi Y, Schlaepfer DD and Cheng SY: Angiopoietin 2 induces glioma cell invasion by stimulating matrix metalloprotease 2 expression through the alphavbeta1 integrin and focal adhesion kinase signaling pathway. Cancer Res 66: 775-783, 2006.

26. Nasarre P, Thomas M, Kruse K, Helfrich I, Wolter V, Deppermann C, Schadendorf D, Thurston G, Fiedler U and Augustin HG: Host-derived angiopoietin-2 affects early stages of tumor development and vessel maturation but is dispensable for later stages of tumor growth. Cancer Res 69: 1324-1333, 2009.

27. Wang HL, Deng CS, Lin J, Pan Dy, Zou Zy and Zhou XY: Expression of angiopoietin-2 is correlated with vascularization and tumor size in human colorectal adenocarcinoma. Tohoku J Exp Med 213: 33-40, 2007.

28. Yancopoulos GD, Davis S, Gale NW, Rudge JS, Wiegand SJ and Holash J: Vascular-specific growth factors and blood vessel formation. Nature 407: 242-248, 2000.

29. Etoh T, Inoue H, Tanaka S, Barnard GF, Kitano S and Mori M: Angiopoietin-2 is related to tumor angiogenesis in gastric carcinoma: Possible in vivo regulation via induction of proteases. Cancer Res 61: 2145-2153, 2001. 